\title{
Review
}

\section{Complete in vitro oogenesis: retrospects and prospects}

\author{
Jun-Jie Wang ${ }^{1}$, Wei Ge ${ }^{1}$, Jing-Cai Liu ${ }^{1}$, Francesca Gioia Klinger ${ }^{2}$, Paul W Dyce ${ }^{3}$, Massimo De Felici ${ }^{\star, 2}$ and Wei Shen ${ }^{\star, 1}$
}

Precise control of mammalian oogenesis has been a traditional focus of reproductive and developmental biology research. Recently, new reports have introduced the possibility of obtaining functional gametes derived in vitro from stem cells. The potential to produce functional gametes from stem cells has exciting applications for regenerative medicine though still remains challenging. In mammalian females ovulation and fertilization is a privilege reserved for a small number of oocytes. In reality the vast majority of oocytes formed from primordial germ cells (PGCs) will undergo apoptosis, or other forms of cell death. Removal occurs during germ cell cyst breakdown and the establishment of the primordial follicle (PF) pool, during the long dormancy at the PF stage, or through follicular atresia prior to reaching the ovulatory stage. A way to solve this limitation could be to produce large numbers of oocytes, in vitro, from stem cells. However, to recapitulate mammalian oogenesis and produce fertilizable oocytes in vitro is a complex process involving several different cell types, precise follicular cell-oocyte reciprocal interactions, a variety of nutrients and combinations of cytokines, and precise growth factors and hormones depending on the developmental stage. In 2016, two papers published by Morohaku et al. and Hikabe et al. reported in vitro procedures that appear to reproduce efficiently these conditions allowing for the production, completely in a dish, of a relatively large number of oocytes that are fertilizable and capable of giving rise to viable offspring in the mouse. The present article offers a critical overview of these results as well as other previous work performed mainly in mouse attempting to reproduce oogenesis completely in vitro and considers some perspectives for the potential to adapt the methods to produce functional human oocytes.

Cell Death and Differentiation (2017) 24, 1845-1852; doi:10.1038/cdd.2017.134; published online 25 August 2017

\section{Facts}

- Complete oogenesis in vitro and obtaining fertilizable oocytes are not only conducive to understanding the regulatory mechanisms of oogenesis, but also to improving the fecundity of mammalian females.

- Recently, reconstituting mouse oogenesis from endogenous PGCs or PGCLCs derived from ES or iPS cells completely in vitro have made significant breakthroughs.

- Among the three main steps involved in mammalian oogenesis in vitro (PGC induction, oocyte differentiation and growth and oocyte maturation/IVF), obtaining oocytes at the secondary follicle stage from PGCs or PGCLCs is the most challenging event during the oocyte differentiation process.

- Successful recapitulation of mouse oogenesis, under completely in vitro conditions, provides a valuable model for studying the mechanisms underlying mammalian oogenesis, particularly for human focused studies.

\section{Open Questions}

- What's responsible for the low efficiency of PGCLCs in producing viable oocytes compared with endogenous PGCs?
- Are there differences between offspring derived from endogenous PGCs and those derived from PGCLCs?

- Is the current system applicable to human stem cell derived oogenesis?

- Will the current system prove to be the ultimate strategy for female infertility treatments?

Due to their unique developmental and regulatory mechanisms, the majority of the mammalian oocyte resource is not fully utilized. For this reason, the generation of functional oocytes in vitro is not only an important target of reproductive research, aiming to identify the underlying regulatory mechanisms of oogenesis, but also a way to optimize the use of the female germ cell pool to improve present assisted reproductive technologies (ART) and to ameliorate female health.

Oocyte in vitro maturation (IVM) and in vitro fertilization (IVF) technologies have been widely used in research and clinical medicine, while reproducing complete oogenesis in vitro and obtaining mature and fertilizable oocytes capable of giving rise to new individuals has remained elusive until very recently. As early as 1971 , Odor and Blandau ${ }^{1}$ reported the first noteworthy results showing that it was possible to obtain a large number of growing mouse and human oocytes by culturing small pieces of fetal ovary in rose chambers for 3-5 weeks

\footnotetext{
${ }^{1}$ Institute of Reproductive Sciences, College of Life Sciences, Qingdao Agricultural University, Qingdao 266109, China; ${ }^{2}$ Department of Biomedicine and Prevention, University of Rome 'Tor Vergata', Rome 00133, Italy and ${ }^{3}$ Department of Animal Sciences, Auburn University, Auburn, AL 36849, USA

${ }^{*}$ Corresponding author: W Shen, Institute of Reproductive Sciences, College of Life Sciences, Qingdao Agricultural University, 700 Changcheng Road, Chengyang, Qingdao 266109, China. Tel: +86 532880302 46; Fax: +86 532860807 73; E-mail: wshen@qau.edu.cn

or M De Felici, Department of Biomedicine and Prevention, University of Rome 'Tor Vergata', Rome 00133, Italy. Tel: +39 0672596156 ; Fax: +39 0672596172 ; E-mail: defelici@uniroma2.it

Received 25.1.17; revised 13.6.17; accepted 20.7.17; Edited by Y Shi; published online 25.8.17
} 

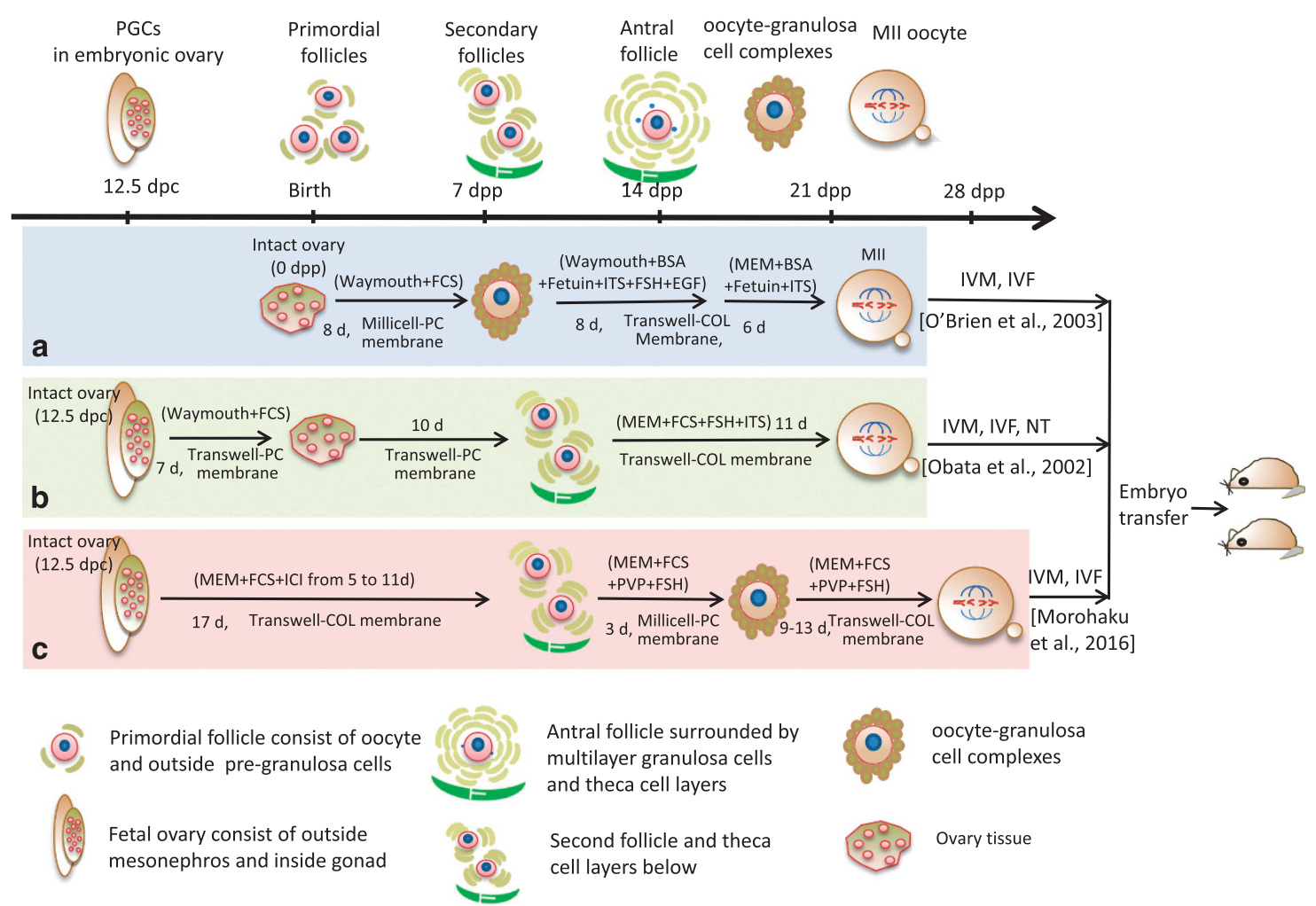

Figure 1 Schematic representation of the main stages of mouse oogenesis (upper drawing) and of the three in vitro culture methods of endogenous germ cells (medial drawings) capable of producing metaphase II (MII) oocytes that after in vitro maturation (IVM) and in vitro fertilization (IVF) or nuclear transfer (NT) generated live offspring; most essential components of the culture media are also reported. The upper drawing displays the process of oogenesis from 12.5 days post-coitum (dpc) and folliculogenesis, including the essential stages of embryonic primordial germ cells (PGCs) at $12.5 \mathrm{dpc}$, primordial follicles at birth, and the formation of secondary and antral follicles at 7 and 14 days post-partum (dpp), development of mature gametes as oocyte-granulosa cell complexes, oocyte meiosis and IVF. The medial figure shows the offspring through in vitro culture with $1 \mathrm{dpp}$ ovary by O'Brien et al. (a) and fetal ovary at $12.5 \mathrm{dpc}$ by Obata et al. (b) and Morohaku et al. (c) The lower panel shows the different cell types annotated

using a modified Eagle's medium containing $10 \%$ heat inactivated horse serum. These oocytes were capable of completing the meiotic prophase with some reaching a diameter of $80-100 \mu \mathrm{m}$, including the zona pellucida, within a multilaminar follicle. After these promising results, many other attempts were made to reconstitute the entire oogenesis process in vitro from follicular oocytes or primordial germ cells (PGCs) (Figure 1 and Table 1). Attempts included using isolated PGCs and culturing on cell monolayers or inside gonadal tissues (pieces of ovarian tissues, ovarian somatic cell aggregates and organotypic cultures). More recently, the possibility of obtaining PGCs from various types of pluripotent stem cells has provided a new cellular input and greatly increased the interest in such studies. However, only very recently two papers from the same research team have reported successful reconstitution of the complete mouse oogenesis process in vitro using the same protocol for all steps except the initial source of the PGCs (endogenous PGCs vs PGC-like cells (PGCLCs) derived from embryonic stem cells (ESCs) and induced pluripotent stem cells (iPSCs)). ${ }^{2,3}$

In the present work, we review the main information and faults of experiments performed mainly in the mouse in the attempt to reconstruct oogenesis in vitro starting from PGCs that preceded these papers and made the extraordinary results achieved by these authors (Figure 1 and Table 1). Moreover, the main improvements introduced by the
Morohaku et al. ${ }^{2}$ in vitro procedures allowing a robust number of oocytes to acquire complete meiotic maturation and the capacity to support embryo development after fertilization, will be discussed. Finally, some perspectives on the potential to transfer such results from mice to humans will be considered.

\section{Outlines of Mouse Oogenesis}

Since the mouse is the species in which the papers by Morohaku et al. and Hikabe et al. were performed and most experimental studies of in vitro oogenesis were carried out, a brief outline of mouse oogenesis follows (Figure 1).

In the mouse embryo, a small number of epiblast cells (about 5-6) are induced to become the PGC precursors (around 6.0 days post-coitum, dpc) by various bone morphogenetic proteins (BMPs), in particular BMP4, secreted by the neighbouring extraembryonic tissues. ${ }^{4}$ These committed cells are then specified and destined to become PGCs while moving to, and allocated in, the allantois $(7.5-8.5 \mathrm{dpc})$. After migration into the gonadal ridges $(9.5-10.5 \mathrm{dpc})$, and some rounds of mitotic proliferation, PGCs differentiate into mitotic oogonia which then enter into meiosis $(13.5-14.5 \mathrm{dpc})$ and become primary oocytes. Since PGC/oogonia mitosis often ends with incomplete cytokinesis, primary oocytes develop as clusters or nests of cells in which daughter cells are joined by intercellular bridges. ${ }^{5}$ Primary oocytes progress to meiotic 
Table 1 Culture systems devised in the attempt to reconstitute various stages of mouse oogenesis in vitro

\begin{tabular}{|c|c|c|c|c|}
\hline Germ cell sources & Culture method & Results & Year & References \\
\hline 12.5 dpc ovary & OC attached on plates & MII & 2009 & 29 \\
\hline 12.5 dpc ovary & OC Transwell membrane on+FC on Transwell-COL membrane+NT & Pups & 2002 & 12 \\
\hline 12.5 dpc ovary & $\begin{array}{l}\text { OC on Transwell-COL membranes+FC on Millicell membrane+FC on } \\
\text { Transwell-COL or Millicell membranes }\end{array}$ & Pups & 2016 & 2 \\
\hline 16.5 dpc ovary & OC on Millicell-PC membrane+ FC culture in droplets & Morulae-blastocysts & 2007 & 45 \\
\hline Primordial follicles & OC on Millicell-PC membrane+FC Transwell-COL membrane & Pups & 1996,2003 & 11,39 \\
\hline $\begin{array}{l}\text { Primary/early } \\
\text { secondary follicles }\end{array}$ & FC in Collagen gels+ on Transwell-COL membrane & Pups & 2013 & 42 \\
\hline $\begin{array}{l}\text { Oocytes from } \\
12-14 \text { dpp ovary }\end{array}$ & Oocytes co-cultured with PAGCs & Morula -blastocysts & 2011 & 46 \\
\hline ES cells & Reconstituted ovaries+transplantation & Offspring & 2012 & 17 \\
\hline ES cells & Reconstituted ovaries+IVDi +IVG & Offspring & 2016 & 3 \\
\hline
\end{tabular}

Abbreviations: COL, collagen; FC, follicles culture; IVDi, in vitro differentiation; IVG, in vitro growth; MII, meiosis II; NT, nuclear transfer; OC, organ culture; PAGCs, preantral granulosa cell

prophase I and arrest at the diplotene stage around (start from $16.5 \mathrm{dpc}$ ) or early after birth. During this period more than onethird of the oocytes degenerate by apoptosis and/or autophagy. ${ }^{6,7}$ At about 4 days post-partum (dpp), the majority of germ cells have disappeared ${ }^{8}$ and the surviving oocytes are individually encircled by pregranulosa cells forming a primordial follicle (PF). In the mouse ovary there is compelling evidence for the existence of two distinct classes of PFs; a first class form that are synchronously activated within a short time window around birth within the ovary medulla and a second class form in the cortex that are gradually activated in adulthood. It was calculated that a minimum of 23 and 47 days is needed for the first and the second classes of PFs, respectively, to reach the antral stage. The first wave of follicles exists in the ovaries for about 3 months and contributes to the onset of puberty and to early fertility. PFs in the ovarian cortex gradually replace the first wave of follicles providing fertility until the end of reproductive life. ${ }^{9}$

The PF pool represents the 'ovarian reserve' from which cohorts of follicles are continuously activated, from birth to menopause, to undergo extensive growth and development as primary and secondary follicles in a process termed folliculogenesis. Oocytes grow from $12-20 \mu \mathrm{m}$ in diameter in primordial and primary follicles, to $70 \mu \mathrm{m}$ in medium size preantral follicles and eventually to a fully grown size of approximately $80 \mu \mathrm{m}$ in large antral follicles. Growing oocytes start to synthesize the zona pellucida glycoproteins, accumulate RNAs and proteins, and are connected through gap junctions to surrounding granulosa cells, which supply them with small metabolites, such as energy substrates, nucleotides and amino acids. During the growing phase oocytes progressively acquire competence to resume meiosis, undergo fertilization and complete preimplantation development. Competence to resume meiosis is not achieved until late in oocyte development and appears correlated with the oocyte diameter ( $\geq 60 \mu \mathrm{m})$, the appearance of a chromatin rim around the nucleolus and cytoskeletal reorganization. ${ }^{10}$ Shortly before ovulation, the meiotic cell cycle is reactivated in the oocytes inside the preovulatory antral follicles and another meiosis II arrest ensues (the metaphase II block); reactivation and completion of meiosis is then dependent upon fertilization. Depending on the strain, an adult female mouse produces from 4 up to 12 fertilizable oocytes every 4-5 days for about 7-8 months.

\section{Key Events For Mouse Oogenesis In Vitro}

Eppig and O'Brien ${ }^{11}$ applied a two-step culture method with isolated newborn mouse ovaries (PF stage) to generate live offspring in 1996. The initial step involved ovarian organ culture and was used to produce preantral follicles, in the second step oocyte-granulosa cell complexes were isolated from the ovarian organ and further cultured to complete oocyte growth and development (Figure 1a). In order to reconstruct the entire mouse oogenesis process in vitro from endogenous PGCs or PGCLCs obtained from ES or iPS cells, numerous exploratory experiments were implemented (Table 1). In 2002 Obata et $a .^{12}$ translocated the nucleus of IVG oocytes that were incapable of resuming meiosis into completely growth oocytes of the normal adult mouse, then the reconstructed oocytes were subjected to IVM and fertilization and resulted in live offspring (Figure 1b). In 2016 a multistep culture procedure was devised by Morohaku et al. ${ }^{2}$ and Hikabe et al., ${ }^{3}$ respectively (Figures 1c and 2). Morohaku and colleagues significantly improved the procedures to obtain secondary follicles from intact embryonic ovary cultures and to produce oocytes able to complete meiotic maturation and support embryo development from cultured oocyte-granulosa cell complexes. Hikabe and colleagues following Morohaku et al. added two steps to the procedures consisting of producing PGCLCs from epiblast like cells (EpiLCs) and reconstructing embryonic ovaries by aggregating PGCLCs with ovarian somatic cells (Figure 2). In this way the authors were able to, for the first time, reconstitute in vitro the entire cycle of the mouse female germ line from embryo to adult. Essentially, these authors combined previously developed procedures and used information obtained by other groups to reproduce, in vitro, distinct stages of oogenesis (Tables 1 and 2).

\section{PGCLCs Induction from EpiLCs In Vitro and their Development into Germ Cells In Vivo}

Culture systems in which PGC specification processes were reconstituted in vitro from the epiblast had been developed in the early 2000s. ${ }^{13-16}$ More recently, PGCLCs were obtained 


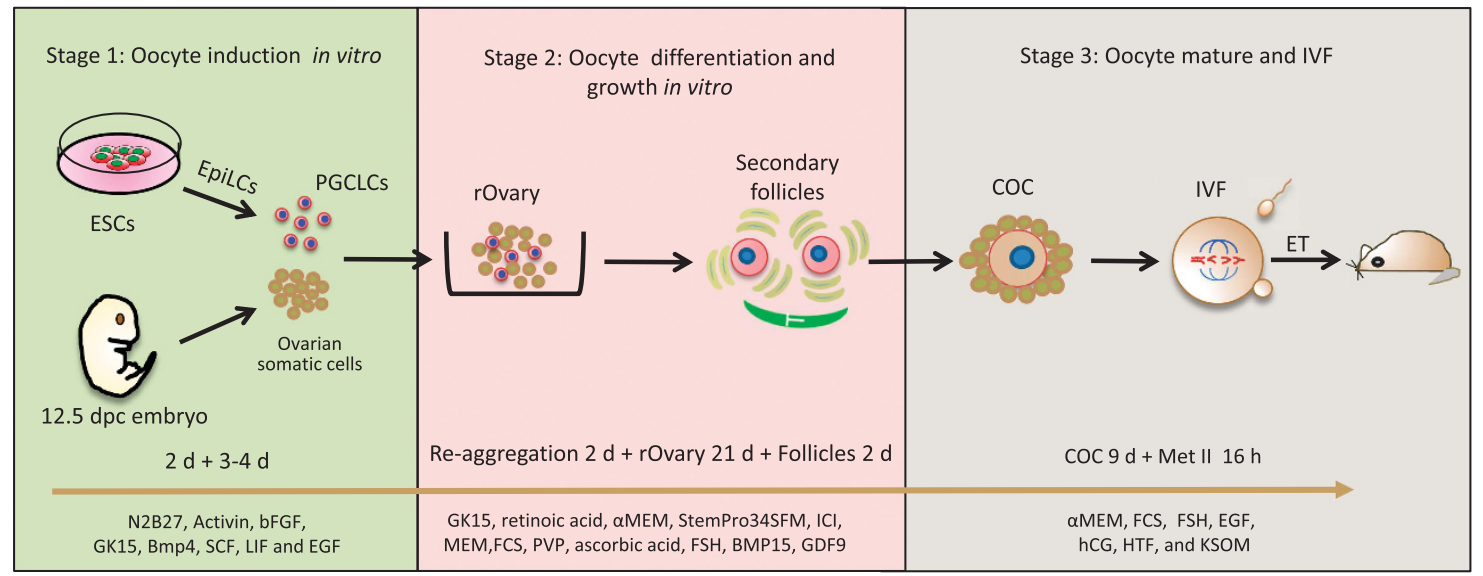

Figure 2 The main steps involved in the procedure of reconstructing the entire mouse oogenesis from exogenous stem cells in vitro (see Hikabe et al. ${ }^{3}$ ). This process was divided into three parts: oocyte induction in vitro (left panel), oocyte differentiation and growth in vitro (middle panel), oocyte maturation and IVF (right panel). Each panel consists of the experimental schematic and in vitro culture details involved in each process

Table 2 Some key factors associated with the development of mouse oocyte and follicle in vitro

\begin{tabular}{|c|c|c|}
\hline Factor/s & Culture method & References \\
\hline $\mathrm{FSH}$ & Essential for follicular survival and development, contributes to granulosa cells proliferation & $44,47-51$ \\
\hline EGF and IGF-I & Have an effect on follicle growth and development and embryo development after fertilization. & $11,52,53$ \\
\hline Activins & Promote antral cavity formation and granulosa cells proliferation, affect follicle growth and development & $35,54-60$ \\
\hline $\mathrm{KL}$ (or SCF) & Promote the oocyte survival and growth & 21 \\
\hline CAMP & $\begin{array}{l}\text { Control of meiotic progression, gap-junctional communication between granulosa cells and oocyte and } \\
\text { antral cavity formation }\end{array}$ & $61-66$ \\
\hline PVP & Maintains the integrity of follicles or COCs in culture & 67,68 \\
\hline Ascorbic acid & Acts likely as antioxidant preventing deleterious action of the free radicals & 69,70 \\
\hline Collagen & $\begin{array}{l}\text { Favours follicle/COC adhesion, stimulates cell proliferation and development and support biological } \\
\text { signalling pathways }\end{array}$ & $3,11,42,43$ \\
\hline ICl182780 & $\begin{array}{l}\text { Antagonist of oestrogen receptor, its addition to the culture medium of ovarian tissue results in a marked } \\
\text { increase of secondary follicle number }\end{array}$ & 2,3 \\
\hline Theca layers & $\begin{array}{l}\text { Their removal exposes COC to the culture medium resulting in considerable improvement of its } \\
\text { development and the acquisition of meiotic maturation by the oocyte }\end{array}$ & 2,3 \\
\hline
\end{tabular}

from ESCs and iPSCs. ${ }^{17-19}$ In such systems, the ESCs/iPSCs were first differentiated into a novel type of cell resembling the post-implantation epiblast called EpiLCs by inducing this status under defined conditions using basic fibroblast growth factor (bFGF) and activin A (ActA). The EpiLCs then efficiently differentiated into PGCLCs in response to BMP4, Kit ligand $(K L)$, leukaemia inhibitory factor (LIF) and epidermal growth factor (EGF). Such PGCLCs were shown to be fully functional, if they were moved from in vitro to in vivo conditions, and appeared capable to differentiate into fertile sperms or mature oocytes; in the first case, upon in vivo transplantation into testicular tubules, in the second, after aggregation with $12.5 \mathrm{dpc}$ ovarian somatic cells to reconstitute an ovary and then transplanting under the ovarian bursa of immunocompromised mice. $^{19}$ In both cases, eggs fertilized with PGCLCderived sperms or eggs-derived from PGCLCs fertilized by sperms from a donor male were showed to be able to give rise to healthy pups that eventually developed to fertile adults. PGCLCs were obtained from epiblast stem cells (EpiSCs) by Hikabe and colleagues using basically the same procedure described above. . $^{3,19}$

\section{Reconstituted Embryonic Ovaries with PGCs and Ovarian Somatic Cells}

Although cultures of isolated PGCs allowed the identification of factors crucial for their survival, proliferation and differentiation such as $\mathrm{KL}$, LIF, bFGF and retinoic acid, ${ }^{20}$ they also showed that germ cells were unable to progress beyond the first stage of meiotic prophase I outside the gonadal microenvironment. Likewise, mouse fetal oocytes stimulated by $\mathrm{KL}$ appeared able to begin the growing phase in the absence of somatic cell support but needed follicular cells to further progress through development. ${ }^{21}$ For this reason, in an attempt to achieve complete oogenesis in vitro, various germ cell-ovarian somatic cell aggregation methods were utilized. Early experiments had shown that aggregation of PGCs with gonadal somatic cells resulted in reconstructed gonads that could be cultured in vitro for a short time to study some aspects of their proliferation and differentiation or transplanted in vivo to achieve complete gametogenesis. In particular, a number of papers showed that ovaries reconstructed by aggregating premeiotic female PGCs with ovarian somatic cells and transplanting under the kidney capsule or the ovarian bursa of 
syngeneic hosts were able to form preantral and antral follicles and develop into meiotic competent and fertilizable oocytes. ${ }^{22-25}$ In this regard, it is important to point out that the developmental stage of the ovary from which PGCs were isolated and the proper synchronization of the germ cellsomatic cell interactions were considered crucial for germ cell survival and completion of oogenesis after transplantation. In fact, Lei et al. ${ }^{22}$ found that in transplanted reconstructed ovaries before $13.5 \mathrm{dpc}$, PGCs completed prophase of meiosis I but did not survive and form follicles. The same results were obtained when $12.5 \mathrm{dpc}$ PGCs were aggregated with somatic cells from fetal ovaries of later developmental stages, competent to form follicles after kidney capsule transplantation or in vitro culture. ${ }^{25}$ In contrast, other papers, reported that aggregates of $12.5 \mathrm{dpc}$ ovarian cells following heterotopic transplanted into adult females were able to produce fertilizable oocytes. ${ }^{23,25}$

\section{Culture of Reconstituted Ovaries}

Female mouse PGCs isolated and aggregated with ovarian somatic cells of the same or other stages were found to enter meiosis and give rise to primary oocytes of $30-35 \mu \mathrm{m}$ diameter. These cells appeared, however, unable to form follicles or to further progress into the growing phase under completely in vitro conditions. ${ }^{22,26,27}$

As far as we know, before the Hikabe and colleagues' paper, $^{3}$ the only attempt to culture PGCLCs in vitro within ovarian somatic cell aggregates was that by Hayashi and Surani. ${ }^{28}$ In this work PGCLCs obtained from EpiSCs were mixed with gonadal cells from $12.5 \mathrm{dpc}$ ovaries and, after centrifugation, the cell suspension was left to aggregate and seeded through the gaps present between small pieces of $12.5 \mathrm{dpc}$ ovaries on transwell inserts (Figure 3a). After 40 days in culture, oocyte-like cells (OLCs) probably derived from PGCLCs were observed although with a very low frequency of around one OLC out of 5000 PGCLCs. Conversely, Hikabe and colleagues aggregated PGCLCs with 12.5 dpc ovaries in a

Transwell-COL membrane

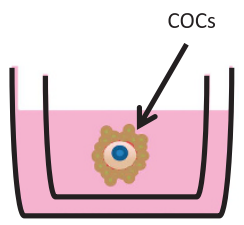

C

Attachment to culture dish

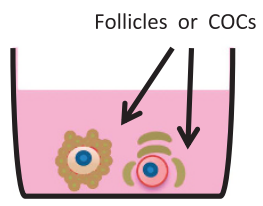

b

Transwell-COL membrane embedded in collagen

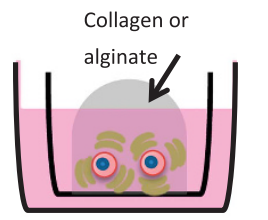

d V-shaped micro-well

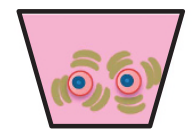

Figure 3 Schematic representation of four culture methods developed for mouse and human primordial/primary follicles or oocytes-cumulus cell complexes (COCs). (a) Eppig and O'Brien; ${ }^{11}$ O'Brien et al. ${ }^{39}$ (mouse). (b) Pangas et al. ${ }^{71}$; Mochida et al. ${ }^{42}$ (mouse). (c) Shen et al., ${ }^{43}$ Cortvrindt et al. ${ }^{44}$ (d) Telfer et al. (human) ${ }^{72,73}$ a low-binding U-bottom 96-well plate (Figures 2 and 3d) for 2 days in serum-free GK15 with knockout serum replacer and the average number of germinal vesicle (GV) stage oocytes per recombinant ovary (consisting of 5000 PGCLCs and 50000 gonadal somatic cells) was $55.1 .^{3}$

The conditions for long-term in vitro culture of reconstructed mouse embryonic ovaries suitable to obtain secondary follicles containing mature GV stage oocytes capable of supporting embryo development after fertilization were not established until the paper by Hikabe et al. ${ }^{3}$ This method was established on the basis of the results obtained in a parallel paper in which the same group was able to reconstruct complete oogenesis in vitro from $12.5 \mathrm{dpc}$ whole ovaries. ${ }^{2}$

Before the Morohaku and colleagues' work, ${ }^{2}$ several attempts were performed in the mouse to produce fertilizable oocytes entirely in vitro by culturing intact or pieces of embryonic ovaries (Figure $1 \mathrm{a}$ and $\mathrm{b}$ and Table 1). The more advanced developmental phases of mouse oogenesis obtained in vitro by culturing intact embryonic ovaries was the preantral and antral follicle stages ${ }^{12,29,30}$ (Figure $1 \mathrm{~b}$ and Table 1). However, GV stage oocytes obtained from such follicles were unable to resume meiosis. Other authors cultured whole or pieces of embryonic ovaries allowing them to attach and spread onto the bottom of the culture dish (Figure 3c). ${ }^{27,31-35}$ In such cultures, mouse PGCs were able to differentiate into primary oocytes that were eventually assembled into primordial or primary follicles. Within these structures, apparently morphologically normal GV stage oocytes grew considerably in volume reaching a maximum diameter of around $65-70 \mu \mathrm{m}$, but did not acquire the capability to resume and complete meiosis. The only exception was reported by Shen's group, ${ }^{35}$ who showed that a small number of such oocytes were able to resume meiosis and be fertilized providing ActA was present in the medium throughout the entire culture time (Table 1).

These experiments pointed out that it was relatively simple to induce the development of female PGCs into primary oocytes that are able to complete meiotic prophase I and be assembled into primordial/primary follicles and almost complete the growing phase inside cultured ovarian tissues in vitro. Moreover, this system allowed the identification of some key factors necessary for oocyte and/or granulosa cell development such as KL, EGF, IGF-1, Activins and others (Table 2). However, they also indicated that almost invariably such oocytes lacked factors necessary to acquire complete meiotic maturation and the capacity to support embryo developmental following fertilization.

\section{Oestrogen Inhibition Favours PF Assembly While Polyvinylpyrrolidone Stabilizes Correct Oocyte- Granulosa Cell Relationships in the Secondary Follicles}

In our view, a major breakthrough of the Morohaku and colleagues studies was to establish in vitro culture conditions that allowed more efficient formation of PFs within the cultured ovaries and the maintenance of the correct interactions between the oocyte and the granulosa cells during the subsequent development of the primary and secondary follicles. ${ }^{2}$ They achieved such important points apparently in a very simple way, by timely adding to the culture medium of ovarian 
tissues an oestrogen receptor antagonist (ICI 182,780, 7a, $17 \beta$-[9-[(4,4,5,5,5-Pentafluoropentyl)sulfinyl]nonyl]estra-1,3,5 (10)-triene-3,17-diol) and a high concentration (2\%) of polyvinylpyrrolidone (PVP), a water soluble polymer made from the monomer $\mathrm{N}$-vinylpyrrolidone.

The reason for adding $\mathrm{ICI}$ was that previous papers clearly showed that reduced concentrations of oestrogens and/or progesterone were essential to promote germ cell cyst breakdown and follicle formation in mice and rats. ${ }^{36,37}$ As a matter of fact, oestrogens are present in the fetal calf serum (FCS), necessary for optimizing the ovary culture, and likely produced by the cultured granulosa cells. Morohaku and colleagues described that the addition of $\mathrm{ICI}$ from day 5 to day 11 of culture of the $12.5 \mathrm{dpc}$ ovary (equivalent to $17.5 \mathrm{dpc}-$ $4 \mathrm{dpp}$, the period of PF assembly in the mouse), resulted in a more than sevenfold increase in the number of secondary follicles isolated from the cultured ovaries on day 17 of culture. Moreover, ICI treatment promoted the formation of a complete laminin layer around individual follicles.

Secondary follicles were mechanically isolated on day 17 of culture since longer culture of the whole ovaries resulted in marked follicle degeneration. These follicles, containing oocytes with a mean diameter of $54.4 \mu \mathrm{m}$, were then cultured for 3 days in minimum essential medium-alpha supplemented with $5 \%$ FCS, $0.1 \mathrm{IU}$ FSH and $2 \%$ PVP on a millicell or transwell-coll membrane. In addition, Hikabe and colleagues added BMP15 and growth differentiation factors 9 (GDF9), two growth factors secreted by the oocytes to stimulate granulosa cell proliferation and steroidogenesis.

The exact role of PVP in this culture step, as well as in the next (see below), is not known. PVP and polyvinylalcohol are frequently added at low concentration (not $>0.4 \%, \mathrm{w} / \mathrm{v}$ ) to medium as a substitute for macromolecules in serum, which provide some colloid osmotic pressure and are useful for preventing loss of oocytes/embryos due to sticking to the glass or plastic surface. Morohaku and colleagues suggest that they add PVP because of its effect on maintaining the integrity of oocytes-cumulus cell complexes (COCs) in a long-term culture found in their previous studies in cattle. ${ }^{2,38}$ In addition to its notable effect on favouring correct oocyte-granulosa cell growth in the secondary follicles, PVP was found to positively affect the expression of genes encoding factors involved in granulosa cell proliferation, such as Bmp6, Bmp15, Kit and Kitl. A plausible explanation of the PVP effect is that it and other beneficial factors were localized within the complexes due to restricted diffusion.

\section{Removal of Follicular Theca and the Presence of PVP are Essential for Complete COC Maturation}

To reconstitute gametogenesis under complete in vitro conditions, Morohaku et al. ${ }^{2}$ recently established a robust protocol to faithfully give rise to viable offspring. Female gonads obtained at $12.5 \mathrm{dpc}$ were cultured for 17 days on transwellcollagen (COL) membranes, after the initial culture for 3 days, the resulting secondary follicles were treated with collagenase to remove theca layers and allow the oocytes to undergo full growth, maturation, fertilization and complete embryo development (Figure 2). It is probable that exposure of COCs directly to the culture medium may promote oocyte development by supporting direct exchange of compounds. Theca removal had been first used to culture mouse COCs in a two-step culture protocol devised by the Eppig group. The result

of this system was the production of oocytes competent to undergo maturation, fertilization and development to live offspring starting from the PFs of newborn mice. ${ }^{11,39}$ Briefly, ovaries were initially isolated from newborn mice and cultured on Millicell-PC membrane inserts (organ culture), for the second step the cultured ovaries were digested with collagenase and DNase to isolate oocyte-granulosa cell complexes and these complexes were cultured for 8 days on transwellCOL membranes (follicle culture).

Subsequent COCs were cultured for another 9-13 days under the same conditions reported above resembling that of Eppig and O'Brien ${ }^{11}$ and O'Brien et al., ${ }^{39}$ but with two main differences: the presence of $5 \%$ FCS and $2 \%$ PVP. At this stage, PVP appeared important for maintaining compact COCs attached to the insert membrane allowing a significantly larger number of complexes to be recovered. Although a direct comparison of the protocols has not been studied, it was likely that in the Eppig and O'Brien protocols the function of FCS and PVP was replaced, at least partially, by BSA, fetuin, EGF and ITS. The possibility to achieve functional oocytes within COCs independent from the formation of antral follicles is a really important point. It provides a solution to the completion of folliculogenesis in vitro, in species producing large preovulatory antral follicles such as humans, which is a problem for this type of culture system. Utilizing these novel culture conditions, the potential problems of nutrient, gas and waste exchange are considerably reduced. As we will discuss below, unlike the antral follicle size, the size and structure of COCs vary little among mammalian species and conditions required for maturation of such complexes in vitro are likely to be quite similar.

The subsequent steps of COC mucification and oocyte maturation to the metaphase II stage and finally IVF and embryo transplantation were performed according to standard procedures. $^{11,39}$

\section{Developmental Competence of Oocytes Grown In Vitro Under Different Experimental Protocols}

Although the papers by Morohaku et al. and Hikabe et al. are so far the only that show the complete reconstituted in vitro mouse oogenesis, it is useful to compare their results to those of the Eppig's group. Their groundbreaking work was the first to report the possibility of producing functional mouse oocytes from PFs capable of giving rise to live offspring after fertilization, entirely in vitro. Actually, it is likely that Morohaku and colleagues and Hikabe and colleagues referred to these works for development of their COC culture system.

By comparing the results obtained by Morohaku et al. and O'Brien et al. (Table 3), it clearly appears that the major difference is in the percentage of oocytes able to mature to the metaphase II (Met II) stage within COCs after FSH and LH stimulation. Interestingly, the competence of the mature Met II oocytes to be fertilized and give rise to live offspring are quite similar. Since the protocol of COC culture is almost the same, and O'Brien and colleagues used neonatal ovary developed 
Table 3 A comparison of different protocols used to produce functional oocytes

\begin{tabular}{lccc}
\hline & Morohaku et al. ${ }^{67}$ & Hikabe et al. & O'Brien et al. ${ }^{39}$ \\
\hline Met II & $84(90)$ & $28.9(-)$ & $44(>90)$ \\
2-cells & $43.5(48.2)$ & $36(-)$ & $43(63)$ \\
Pups & $27(59.7)$ & $3.5(61.7)$ & $5(16.5)$ \\
\hline
\end{tabular}

Values represent \%; in parenthesis the values of control in vivo matured oocytes; ' - ' means no available data

in vivo, the reason for such a difference must be in some critical aspect of oocyte development, performed in vitro in both cases, when PF are assembled and the folliculogenesis is activated up to the secondary follicle stage. In fact, O'Brien and colleagues cultured for 8 days newborn ovaries basically only in Waymouth medium supplemented with $10 \%$ FCS, while Morohaku and colleagues added the oestrogen receptor antagonist (ICl182780) and PVP, likely indispensable to more closely recapitulate the physiological ovary development at this stage. However, in the Hikabe paper, the same protocol used in Morohaku and colleagues appears much less efficient in producing mature oocytes and pups when applied to PGCLCs, which may be caused by the deficiency of the current PGCLCs differentiation system, either at the transcriptional or epigenetic level.

In all these works, the timing of the in vitro culture parallels that of mouse oogenesis characterized by a prenatal development of 18-19 days and a first wave of folliculogenesis starting around birth and producing antral follicles after about 3 weeks. All procedures were of course able to produce only one wave of mature oocytes since the cyclic nature of oogenesis could not be reproduced using such systems. Noteworthy, Morohaku and colleagues reported that a maximum of seven pups were obtained from a single gonad, which is comparable with the number seen in a natural cycle in mice.

\section{Prospects}

The procedures discussed above developed in the mouse raise the possibility of growing human oocytes to maturity using a similar in vitro culture system. The potential of such a system with regard to improving human fertility would be great. It would clearly be of benefit in basic physiological studies on human oogenesis. It could be used to test the effect of toxicological substances on oocyte and follicle maturation. Eventually, such a system could provide a source of oocytes for ARTs. Noteworthy, Morohaku and colleagues reported that their procedure was able to produce functional oocytes also from cryopreserved fetal mouse gonads, a common practise in ARTs.

The potential to generate human PGCLCs from ES and iPS cells ${ }^{40}$ then growing functional oocytes, ${ }^{41}$ using procedures quite similar to that used in the mouse is probable due to the fact that the size and structure of COCs vary little among mammalian species. It is likely that the systems will have to be modified for culturing complexes from species other than mouse, in particular to restrict the migration of granulosa cells away from the oocytes, a problem apparently solved in mouse and bovine by PVP. The maintenance of the association between granulosa cells and the oocyte is essential for oocyte growth and development. On the contrary, the apparent asynchrony of PGC/oogonia proliferation and entering into meiosis together with a lack of information about how these processes are regulated in the human fetal ovary, and the scant knowledge of the mechanisms of PF assembly and timing of transition from primordial to primary and secondary follicle stage represent critical points. In the case of using PGCLCs, another limitation is the requirement of gonadal somatic cells for reconstructing the embryonic ovary. As suggested by Hikabe and colleagues to overcome this issue gonadal somatic cell-like cells could be derived from ES or iPS cells. This is a critical step that will need to be addressed to ethically utilize a similar system for human reproduction.

For this reason, the first step towards adapting a culture system for use in humans is to acquire more information about the critical points reported above, and at the same time use animal models with oogenesis characteristics more similar to humans and with an abundant supply of materials. Repeating these studies in non-human primates would be beneficial in elucidating the likelihood of transitioning the findings to humans.

\section{Conflict of Interest}

The authors declare no conflict of interest.

Acknowledgements. This work was supported by National Natural Science Foundation of China (31471346 and 31671554) and National Basic Research Program of China (973 Program, 2012CB944401).

1. Odor DL, Blandau RJ. Organ cultures of fetal mouse ovaries. I. Light microscopic structure. Am J Anat 1971; 131: 387-414

2. Morohaku K, Tanimoto R, Sasaki K, Kawahara-Miki R, Kono T, Hayashi K et al. Complete in vitro generation of fertile oocytes from mouse primordial germ cells. Proc Natl Acad Sci USA 2016; 113: 9021-9026.

3. Hikabe O, Hamazaki N, Nagamatsu G, Obata Y, Hirao Y, Hamada N et al. Reconstitution in vitro of the entire cycle of the mouse female germ line. Nature 2016; 539: 299-303.

4. Wear HM, McPike MJ, Watanabe KH. From primordial germ cells to primordial follicles: a review and visual representation of early ovarian development in mice. J Ovarian Res 2016; 9: 36.

5. Pepling ME. From primordial germ cell to primordial follicle: mammalian female germ cell development. Genesis 2006; 44: 622-632.

6. Lobascio AM, Klinger FG, Scaldaferri ML, Farini D, De Felici M. Analysis of programmed cell death in mouse fetal oocytes. Reproduction 2007; 134: 241-252.

7. De Felici M, Klinger FG, Farini D, Scaldaferri ML, Iona S, Lobascio M. Establishment of oocyte population in the fetal ovary: primordial germ cell proliferation and oocyte programmed cell death. Reprod Biomed Online 2005; 10: 182-191.

8. Pepling ME. Follicular assembly: mechanisms of action. Reproduction 2012; 143: 139.

9. Zheng W, Zhang H, Gorre N, Risal S, Shen Y, Liu K. Two classes of ovarian primordial follicles exhibit distinct developmental dynamics and physiological functions. Hum Mol Genet 2014; 23: 920-928

10. Coticchio G, Albertini DF, Santis LD. Oogenesis. Springer: London, 2013.

11. Eppig JJ, O'Brien MJ. Development in vitro of mouse oocytes from primordial follicles. Biol Reprod 1996; 54: 197-207.

12. Obata $Y$, Kono T, Hatada I. Gene silencing: maturation of mouse fetal germ cells in vitro. Nature 2002; 418: 497.

13. Ohinata $Y$, Ohta H, Shigeta M, Yamanaka K, Wakayama T, Saitou M. A signaling principle for the specification of the germ cell lineage in mice. Cell 2009; 137: 571-584.

14. Pesce M, Klinger FG, De FM. Derivation in culture of primordial germ cells from cells of the mouse epiblast: phenotypic induction and growth control by Bmp4 signalling. Mech Dev 2002; 112: 15-24.

15. Yoshimizu T, Obinata M, Matsui Y. Stage-specific tissue and cell interactions play key roles in mouse germ cell specification. Development 2001; 128: 481-490.

16. Ying $Y, Q i X, Z$ hao $G Q$. Induction of primordial germ cells from murine epiblasts by synergistic action of BMP4 and BMP8B signaling pathways. Proc Natl Acad Sci USA 2001; 98: 7858.

17. Hayashi K, Ogushi S, Kurimoto K, Shimamoto S, Ohta H, Saitou M. Offspring from oocytes derived from in vitro primordial germ cell-like cells in mice. Science 2012; 338: 971-975.

18. Hayashi K, Ohta H, Kurimoto K, Aramaki S, Saitou M. Reconstitution of the mouse germ cell specification pathway in culture by pluripotent stem cells. Cell 2011; 146: 519-532.

19. Hayashi K, Saitou M. Generation of eggs from mouse embryonic stem cells and induced pluripotent stem cells. Nat Protoc 2013; 8: 1513-1524. 
20. De FM. Primordial germ cell biology at the beginning of the XXI century. Int J Dev Biol 2009; 53: 891-894.

21. Klinger FG, De Felici M. In vitro development of growing oocytes from fetal mouse oocytes: stagespecific regulation by stem cell factor and granulosa cells. Dev Biol 2002; 244 85-95.

22. Lei L, Zhang H, Jin S, Wang F, Fu M, Wang $\mathrm{H}$ et al. Stage-specific germ-somatic cell interaction directs the primordial folliculogenesis in mouse fetal ovaries. J Cell Physiol 2006; 208: 640-647.

23. Hayama T, Yamaguchi T, Katoitoh M, Hamanaka S, Kawarai M, Sanbo M et al. Generation of mouse functional oocytes in rat by xeno-ectopic transplantation of primordial germ cells1. Biol Reprod 2014; 91: 89-89.

24. Nicholas CR, Haston KM, Grewall AK, Longacre TA, Reijo Pera RA. Transplantation directs oocyte maturation from embryonic stem cells and provides a therapeutic strategy for female infertility. Hum Mol Genet 2009; 18: 4376-4389.

25. Hashimoto K, Noguchi M, Nakatsuji N. Mouse offspring derived from fetal ovaries or reaggregates which were cultured and transplanted into adult females. Dev Growth Differ 1992; 34: 233-238.

26. De FM, Dolci S. Cellular interactions of mouse fetal germ cells in in vitro systems. Curr Top Dev Biol 1987; 23: 147-162.

27. McLaren A, Buehr M. Development of mouse germ cells in cultures of fetal gonads. Cell Differ Dev 1990; 31: 185-195.

28. Hayashi K, Surani MA. Self-renewing epiblast stem cells exhibit continual delineation of germ cells with epigenetic reprogramming in vitro. Development (Cambridge, England) 2009; 136: 3549-3556.

29. Dong HS, Li L, Song ZH, Tang J, Xu B, Zhai XW et al. Premeiotic fetal murine germ cells cultured in vitro form typical oocyte-like cells but do not progress through meiosis. Theriogenology 2009; 72: 219-231.

30. Song Z, Min L, Pan Q, Shi Q, Shen W. Maternal imprinting during mouse oocyte growth in vivo and in vitro. Biochem Biophys Res Commun 2009; 387: 800-805.

31. Pesce M, Cerrito MG, Travia G, Russo MA, De FM. In vitro development of growing oocytes from fetal and early postnatal mouse ovaries. Int J Dev Biol 1996; 40: (Suppl 1): 229S-230S.

32. Byskov AG, Guoliang X, Andersen CY. The cortex-medulla oocyte growth pattern is organized during fetal life: an in-vitro study of the mouse ovary. Mol Hum Reprod 1997; 3: 795-800.

33. Motohashi HH, Kada H, Sato K. Developmental and ultrastructural characteristics of mouse oocytes grown in vitro from primordial germ cells. Hum Cell 2004; 17: 67-74.

34. Leesmurdock DJ, Lau HT, Castrillon DH, De FM, Walsh CP. DNA methyltransferase loading, but not de novo methylation, is an oocyte-autonomous process stimulated by SCF signalling. Dev Biol 2008; 321: 238-250.

35. Zhang ZP, Liang GJ, Zhang XF, Zhang GL, Chao HH, Li L et al. Growth of mouse oocytes to maturity from premeiotic germ cells in vitro. PLOS ONE 2012; 7: e41771.

36. Chen $\mathrm{Y}$, Breen K, Pepling ME. Estrogen can signal through multiple pathways to regulate oocyte cyst breakdown and primordial follicle assembly in the neonatal mouse ovary. $J$ Endocrinol 2009; 202: 407-417.

37. Kezele P, Skinner MK. Regulation of ovarian primordial follicle assembly and development by estrogen and progesterone: endocrine model of follicle assembly. Endocrinology 2003; 144: 3329-3337.

38. Hirao Y, Naruse K, Kaneda M, Somfai T, Iga K, Shimizu M et al. Production of fertile offspring from oocytes grown in vitro by nuclear transfer in cattle. Biol Reprod 2013; 89: 57.

39. O'Brien MJ, Pendola JK, Eppig JJ. A revised protocol for in vitro development of mouse oocytes from primordial follicles dramatically improves their developmental competence. Biol Reprod 2003; 68: 1682-1686.

40. De FM. The Formation and Migration of Primordial Germ Cells in Mouse and Man. Results Probl Cell Differ 2016; 58: 23-46.

41. Ge W, Chen C, De FM, Shen W. In vitro differentiation of germ cells from stem cells: a comparison between primordial germ cells and in vitro derived primordial germ cell-like cells. Cell Death Dis 2015; 6: e1906.

42. Mochida N, Akatani-Hasegawa A, Saka K, Ogino M, Hosoda Y, Wada R et al. Live births from isolated primary/early secondary follicles following a multistep culture without organ culture in mice. Reproduction 2013; 146: 37-47.

43. Shen W, Zhang D, Qing T, Cheng J, Bai Z, Shi Y et al. Live offspring produced by mouse oocytes derived from premeiotic fetal germ cells. Biol Reprod 2006; 75: 615-623.

44. Cortvrindt R, Smitz J, Van Steirteghem AC. In-vitro maturation, fertilization and embryo development of immature oocytes from early preantral follicles from prepuberal mice in a simplified culture system. Hum Reprod 1996; 11: 2656-2666.

45. Shen W, Li L, Bai Z, Pan Q, Ding M, Deng H. In vitro development of mouse fetal germ cells into mature oocytes. Reproduction 2007; 134: 223-231.

46. Li Z, Zhang P, Zhang Z, Pan B, Chao H, Li L et al. A co-culture system with preantral follicular granulosa cells in vitro induces meiotic maturation of immature oocytes. Histochem Cell Biol 2011; 135: 513-522.

47. Nayudu PL, Osborn SM. Factors influencing the rate of preantral and antral growth of mouse ovarian follicles in vitro. J Reprod Fertil 1992; 95: 349-362.

48. Adriaens I, Cortvrindt R, Smitz J. Differential FSH exposure in preantral follicle culture has marked effects on folliculogenesis and oocyte developmental competence. Hum Reprod 2004; 19: 398-408.

49. Baker SJ, Srsen V, Lapping R, Spears N. Combined effect of follicle-follicle interactions and declining follicle-stimulating hormone on murine follicle health in vitro. Biol Reprod 2001; 65: 1304-1310.
50. Spears N, Murray AA, Allison V, Boland NI, Gosden RG. Role of gonadotrophins and ovarian steroids in the development of mouse follicles in vitro. J Reprod Fertil 1998; 113: 19-26.

51. Cortvrindt R, Smitz J, Van Steirteghem AC. Assessment of the need for follicle stimulating hormone in early preantral mouse follicle culture in vitro. Hum Reprod 1997; 12: 759-768.

52. Demeestere I, Gervy C, Centner J, Devreker F, Englert Y, Delbaere A. Effect of insulin-like growth factor-I during preantral follicular culture on steroidogenesis, in vitro oocyte maturation, and embryo development in mice. Biol Reprod 2004; 70: 1664-1669.

53. Zhou H, Zhang Y. Effect of growth factors on in vitro development of caprine preantral follicle oocytes. Anim Reprod Sci 2005; 90: 265-272.

54. Zhao J, Taverne MA, van der Weijden GC, Bevers MM, van den Hurk R. Effect of activin A on in vitro development of rat preantral follicles and localization of activin $A$ and activin receptor II. Biol Reprod 2001; 65: 967-977.

55. Findlay JK. An update on the roles of inhibin, activin, and follistatin as local regulators of folliculogenesis. Biol Reprod 1993; 48: 15-23.

56. Cossigny DA, Findlay JK, Drummond AE. The effects of FSH and activin A on follicle development in vitro. Reproduction 2012; 143: 221-229.

57. Kipp JL, Golebiowski A, Rodriguez G, Demczuk M, Kilen SM, Mayo KE. Gene expression profiling reveals Cyp26b1 to be an activin regulated gene involved in ovarian granulosa cell proliferation. Endocrinology 2011; 152: 303-312.

58. McLaughlin M, Bromfield JJ, Albertini DF, Telfer EE. Activin promotes follicular integrity and oogenesis in cultured pre-antral bovine follicles. Mol Hum Reprod 2010; 16: 644-653.

59. Bristol-Gould SK, Kreeger PK, Selkirk CG, Kilen SM, Cook RW, Kipp JL et al. Postnatal regulation of germ cells by activin: the establishment of the initial follicle pool. Dev Biol 2006; 298: 132-148.

60. Drummond AE, Dyson M, Mercer JE, Findlay JK. Differential responses of post-natal rat ovarian cells to FSH and activin. Mol Cell Endocrinol 1996; 122: 21-32.

61. Eppig JJ. Maintenance of meiotic arrest and the induction of oocyte maturation in mouse oocyte-granulosa cell complexes developed in vitro from preantral follicles. Biol Reprod 1991; 45: 824-830.

62. Carroll J, Whittingham DG, Wood MJ. Effect of dibutyryl cyclic adenosine monophosphate on granulosa cell proliferation, oocyte growth and meiotic maturation in isolated mouse primary ovarian follicles cultured in collagen gels. J Reprod Fertil 1991; 92: 197-207.

63. Thomas RE, Armstrong DT, Gilchrist RB. Bovine cumulus cell-oocyte gap junctional communication during in vitro maturation in response to manipulation of cell-specific cyclic adenosine 3',5'-monophosophate levels. Biol Reprod 2004; 70: 548-556.

64. Webb RJ, Marshall F, Swann K, Carroll J. Follicle-stimulating hormone induces a gap junction-dependent dynamic change in [CAMP] and protein kinase a in mammalian oocytes. Dev Biol 2002; 246: 441-454.

65. Bornslaeger EA, Schultz RM. Regulation of mouse oocyte maturation: effect of elevating cumulus cell cAMP on oocyte cAMP levels. Biol Reprod 1985; 33: 698-704.

66. Shu YM, Zeng HT, Ren Z, Zhuang GL, Liang XY, Shen HW et al. Effects of cilostamide and forskolin on the meiotic resumption and embryonic development of immature human oocytes. Hum Reprod 2008; 23: 504-513.

67. Morohaku K, Hirao Y, Obata Y. Developmental competence of oocytes grown in vitro: has it peaked already? J Reprod Dev 2016; 62: 1-5.

68. Hirao $\mathrm{Y}$, Itoh T, Shimizu M, Iga $\mathrm{K}$, Aoyagi $\mathrm{K}$, Kobayashi $\mathrm{M}$ et al. In vitro growth and development of bovine oocyte-granulosa cell complexes on the flat substratum: effects of high polyvinylpyrrolidone concentration in culture medium. Biol Reprod 2004; 70: 83-91.

69. Rossetto R, Lima-Verde IB, Matos MH, Saraiva MV, Martins FS, Faustino LR et al. Interaction between ascorbic acid and follicle-stimulating hormone maintains follicular viability after long-term in vitro culture of caprine preantral follicles. Domest Anim Endocrinol 2009; 37: 112-123.

70. Thomas FH, Leask R, Srsen V, Riley SC, Spears N, Telfer EE. Effect of ascorbic acid on health and morphology of bovine preantral follicles during long-term culture. Reproduction 2001; 122: 487-495.

71. Pangas SA, Saudye H, Shea LD, Woodruff TK. Novel approach for the three-dimensional culture of granulosa cell-oocyte complexes. Tissue Eng 2003; 9: 1013-1021.

72. Telfer EE, McLaughlin M, Ding C, Thong KJ. A two-step serum-free culture system supports development of human oocytes from primordial follicles in the presence of activin. Hum Reprod 2008; 23: 1151-1158.

73. Telfer EE, Zelinski MB. Ovarian follicle culture: advances and challenges for human and nonhuman primates. Fertil Steril 2013, 99: 1523-1533.

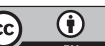

This work is licensed under a Creative Commons Attribution 4.0 International License. The images or other third party material in this article are included in the article's Creative Commons license, unless indicated otherwise in the credit line; if the material is not included under the Creative Commons license, users will need to obtain permission from the license holder to reproduce the material. To view a copy of this license, visit http:/l creativecommons.org/licenses/by/4.0/

(C) The Author(s) 2017 